Received: 2018.10.23 Accepted: 2018.12.21 Published: 2019.03.16

\title{
Oxacillin-Induced Drug Reaction with Eosinophilia and Systemic Symptoms (DRESS)
}

Authors' Contribution: Study Design A Data Collection B Statistical Analysis C Data Interpretation D Manuscript Preparation E Literature Search F Funds Collection G
ABCDEF Alexis Sharpe

ABCDEF Bashar M. Mourad

ABCDEF Chase J. Hardwick

ABCDEF Theresa Reilly

ABCDEF Ezra Dweck

ABCDEF Eric Bondarsky
Corresponding Author: Conflict of interest:
Alexis Sharpe, e-mail: sharpe.alexis@gmail.com

None declared
Department of Pulmonary and Critical Care, NYU Langone, New York City, NY, U.S.A.

Patient: Male, $\mathbf{5 2}$

Final Diagnosis: Drug reaction with eosinophilia and systemic symptoms

Symptoms: Rash

Medication: Oxacillin

Clinical Procedure: -

Specialty: Critical Care Medicine

Objective:

Background:

Case Report:

Conclusions:

MeSH Keywords:

Full-text PDF:

\section{Rare disease}

Drug reaction with eosinophilia and systemic symptoms (DRESS) is an idiosyncratic life-threatening reaction comprised of fevers, rash, and leukocytosis with eosinophilia. Though characteristically associated with leukocytosis, there are rare case reports of DRESS-induced agranulocytosis. DRESS is most frequently caused by antiepileptic medications; however, it has very rarely been reported in relation to oxacillin. We describe a case of oxacillin-induced DRESS associated with agranulocytosis.

A 52-year-old male was admitted for an epidural abscess secondary to oxacillin-sensitive Staphylococcus aureus, for which an extended course of oxacillin and rifampin was initiated. On day 22 of therapy, the patient developed a fever of $38.7^{\circ} \mathrm{C}\left(101.6^{\circ} \mathrm{F}\right)$ with rigors. His complete blood cell count revealed new leukopenia $\left(1.8 \times 10^{3} / \mathrm{uL}\right)$ with $16 \%$ eosinophils and $3 \%$ atypical lymphocytes. Antibiotics were transitioned from oxacillin and rifampin to vancomycin, cefepime, and rifampin for presumed sepsis of unclear etiology. On day 23 , he was noted to have a pruritic erythematous blanching papular rash on his chest, trunk, neck, and left upper extremity. Infectious workup was unrevealing, and his fever curve up-trended to $39.3^{\circ} \mathrm{C}\left(102.7^{\circ} \mathrm{F}\right)$ with no clinical improvement on broad-spectrum antimicrobials, suggestive of a non-infectious etiology of his rash and fevers. His rash evolved into confluent red patches, and eosinophilia rose to $21 \%$, which was concerning for a drug reaction. His RegiSCAR score was calculated to be 6 , consistent with definite DRESS. Leukopenia resolved $\left(6.3 \times 10^{3} / \mathrm{uL}\right) 4$ days after discontinuing oxacillin. His epidural abscess was ultimately treated with daptomycin, and DRESS was managed supportively with antihistamines and triamcinolone cream.

We highlight this case because of the rarity of DRESS with agranulocytosis related to oxacillin. Beta-lactam antibiotics are widely used, and while DRESS is an uncommon condition, clinicians should consider this diagnosis when managing patients with fevers, leukopenia, and rash.

Agranulocytosis • Drug Hypersensitivity Syndrome - Drug-Related Side Effects and Adverse Reactions - Oxacillin

https://www.amjcaserep.com/abstract/index/idArt/913748 


\section{Background}

Drug reaction with eosinophilia and systemic symptoms (DRESS) was first identified in the 1920s as a severe drug reaction to an anti-epileptic medication, nirvanol [1]. Since that time, DRESS has become known as an idiosyncratic life-threatening reaction comprised of fevers, rash, and leukocytosis with eosinophilia. Though a relatively rare syndrome with an incidence of 0.9 affected per 100000 patients, DRESS can lead to severe multi-organ system dysfunction, and in some cases even death [2]. A review of 117 cases of DRESS identified anti-epileptics, allopurinol, and the antimicrobials sulfonamide and dapsone as culprits in two-thirds of cases [3]. Oxacillin, however, has not been cited in the list of more than 50 offending drugs that have been implicated in DRESS $[4,5]$. In our review of the literature, we found extremely rare cases of DRESS attributed to oxacillin [6]; we report here the first case of DRESS due to oxacillin with agranulocytosis.

\section{Case Report}

A 52-year-old Caucasian male was admitted to an academic New York City hospital with complaints of lower extremity pain. Past medical history was significant for type 2 diabetes mellitus, obesity, and osteoarthritis. Prior to admission, the patient reported taking aspirin $81 \mathrm{mg}$ daily, metformin $500 \mathrm{mg}$ 2 times daily with meals, naproxen $500 \mathrm{mg} 2$ times daily with meals, and cyclobenzaprine $10 \mathrm{mg} 3$ times daily as needed. He resided in the United States, his country of origin, and denied any recent travel. Upon admission, he was found to have an epidural abscess. Intra-operative cultures grew Staphylococcus aureus, which was sensitive to oxacillin. He was initiated on an extended course of oxacillin and rifampin for treatment.

On day 22 of therapy, the patient began to develop fevers with maximum temperature $38.7^{\circ} \mathrm{C}\left(101.6^{\circ} \mathrm{F}\right)$ in association with rigors. Physical examination at the time of fever onset was notable for tachycardia, rigors, and apparent discomfort. There was no diaphoresis, and the skin was noted to be warm and dry, without the evidence of a rash or lymphadenopathy. Preliminary workup revealed a new leukopenia $\left(1.8 \times 10^{3} / \mathrm{uL}\right)$ with $16 \%$ eosinophils and $3 \%$ atypical lymphocytes. Given concern for sepsis of unclear etiology in a healthcare system exposed patient, antibiotics were transitioned from oxacillin and rifampin to vancomycin, cefepime, and rifampin for broader antimicrobial coverage.

Infectious workup, however, was unrevealing; blood and urine cultures revealed no growth of organisms, chest $\mathrm{x}$-ray did not show evidence of pulmonary pathology, magnetic resonance imaging (MRI) of the spine revealed no active infectious process, and pro-calcitonin was not significantly elevated $(1.25 \mathrm{ng} / \mathrm{mL})$.

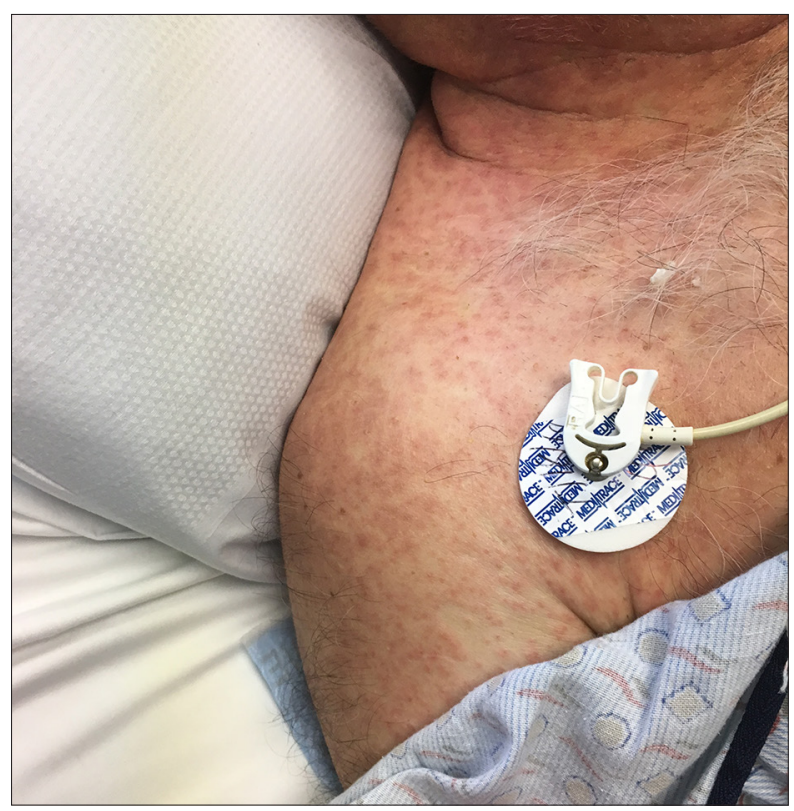

Figure 1. Erythematous rash on chest, neck, and upper extremity.

Human immunodeficiency virus (HIV) testing from the time of admission was negative. Autoimmune workup was notable for a recent negative nuclear antibody, rheumatoid factor, and cyclic citrullinated antibody but did reveal an elevated c-reactive protein $(43 \mathrm{mg} / \mathrm{L})$ and elevated erythrocyte sedimentation rate ( $98 \mathrm{~mm} /$ hour) on the day of fever onset. Other laboratory tests, including renal and hepatic function tests, remained within normal limits.

Despite broad antimicrobial coverage, his fevers trended upwards to a maximum of $39.3^{\circ} \mathrm{C}\left(102.7^{\circ} \mathrm{F}\right)$, and his lack of clinical improvement was suggestive of a non-infectious etiology of his clinical presentation. On day 23 of antibiotics, the patient developed a pruritic, erythematous, blanching, papular rash on his chest, trunk, neck, and left upper extremity (Figure 1), which evolved into confluent red patches (Figure 2). Eosinophilia rose to $21 \%$, which, in the setting of fevers and rash, was concerning for a drug reaction. His RegiSCAR score was calculated to be 6 , which was consistent with definite DRESS. Dermatology was consulted, but a skin biopsy was deferred due to a high probability of DRESS obviating need for pathologic confirmation. His leukopenia resolved 4 days after transitioning from oxacillin to vancomycin and cefepime $\left(6.3 \times 10^{3} / \mathrm{uL}\right)$, highly suggestive of oxacillin as the precipitant.

DRESS was managed supportively with loratadine $10 \mathrm{mg}$ daily and triamcinolone $0.1 \%$ cream twice daily. Given a normal creatinine and hepatic enzyme panel, the decision was made to withhold treatment with systemic corticosteroids, as it was felt that the associated risks of a prolonged and slow steroid taper outweighed the potential benefit in our patient. 


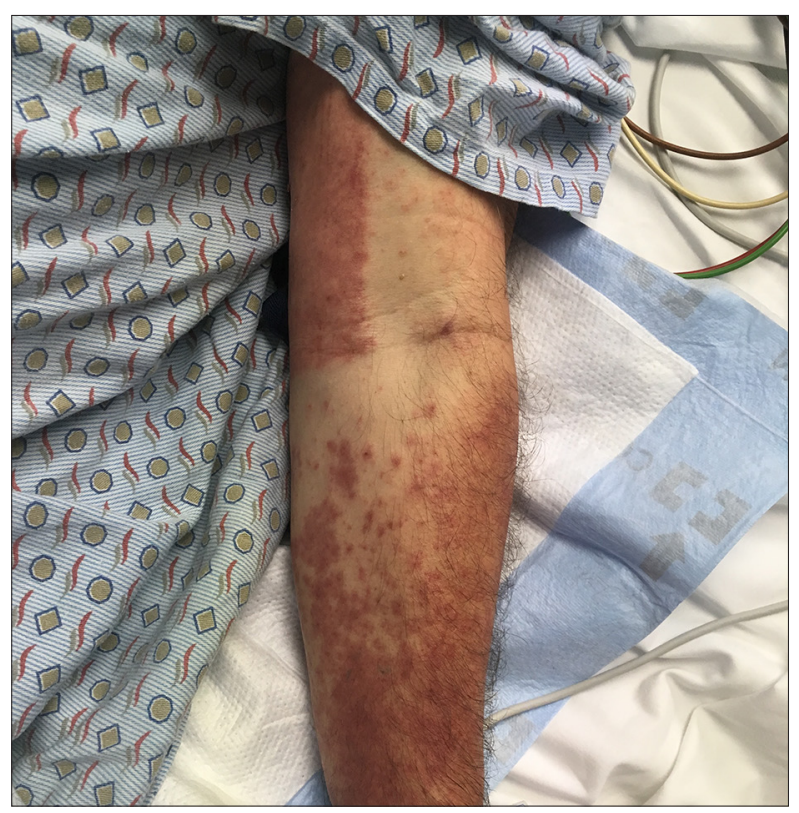

Figure 2. Confluent red patch on upper extremity.

His rash resolved and inflammatory markers down-trended. His epidural abscess ultimately was treated with an extended course of daptomycin.

\section{Discussion}

The constellation of signs and symptoms in DRESS typically includes morbilliform skin rash, fevers, eosinophilia, and lymphadenopathy. Although our patient did not have any solid organ involvement, most patients also suffer from single internal organ involvement, the vast majority of which develop hepatitis [7]. Some cases have been described with multiple organ involvement [8]. While retrospective reviews have identified fever, malaise, and skin eruptions as the most common initial symptoms, this triad is not invariably present [9]. Findings that are most suggestive of DRESS include rash involving greater than $50 \%$ of body surface area and/or 2 of the following: facial edema, infiltrated lesions, scaling, or purpura [3]. Given the heterogeneity of presentation and latency period of a median interval of 22 days from administration of the inciting agent, a definitive diagnosis may be difficult to establish [3].

Clinical features also may vary depending on the inciting agent. Allopurinol, for example, has been associated with a higher incidence of renal involvement and higher eosinophilia [10,11]. Antiepileptics, such as carbamazepine and phenytoin, have rarely been reported to be associated with agranulocytosis [12,13]. Our patient presented with leukopenia and agranulocytosis, which was first noted at the time of onset of fevers and resolved with resolution of DRESS. Other reports of DRESS with agranulocytosis have occurred in the setting of allopurinol and sulfasalazine [13]. Although these aforementioned case reports did reveal atypical lymphocytes, they were not associated with eosinophilia, which our patient did exhibit.

There is only 1 other reported case of DRESS related to oxacillin use, and this is the first reported case of oxacillin associated with agranulocytosis in the setting of DRESS. The previous case of DRESS due to oxacillin, reported in 2015, described a 9-year-old female treated with oxacillin for an abscess due to Staphylococcus aureus. She presented with rash, eosinophilia, and elevated liver function tests on day 25 of treatment; DRESS was confirmed by skin biopsy [6]. This aforementioned case differs from our case reported here, as it was not associated with agranulocytosis, however, both cases present with rash and eosinophilia within a similar time course after the initiation of oxacillin to treat an infection due to Staphylococcus aureus.

Scoring systems such as RegiSCAR have been validated to classify DRESS as possible, probable, or definite; however, some data used in these classification scales are only available later in the disease course, thus limiting their utility in real-time diagnosis [3]. Our patient received a RegiSCAR score of 6 , based on fever, atypical lymphocytes, eosinophilia, skin rash extending over $50 \%$ of body with edema, purpura and scaling, organ involvement as evidenced by bone marrow suppression, resolution delay greater than 15 days, and at least 3 biological investigations done and negative to exclude alternative diagnosis, however, data such as time until resolution were retrospective in nature [14]. As there is no single real-time diagnostic test, the DRESS diagnosis is made by evidence of this constellations of symptoms after autoimmune, infectious, re-activation of EBV and HHV6, and neoplastic processes have been excluded.

The mainstay of treatment of DRESS is removal of the offending agent, supportive management and avoidance of new medications. Though no randomized controlled trials have evaluated the efficacy of high potency corticosteroids, these medications have been used for relief of pruritus and skin inflammation anecdotally. In patients without severe organ involvement, observational data have shown no benefit of systemic compared to topical corticosteroids and in fact favor topical therapies due to lower complication rates $[15,16]$. Systemic steroids are suggested for liver or kidney involvement. For patients whose disease course is refractory to steroids, there is some evidence suggesting cyclosporine results in significant clinical improvement [17], whereas the evidence for intravenous immunoglobulin remains mixed $[18,19]$.

\section{Conclusions}

DRESS is a rare adverse reaction, which, while most frequently associated with anti-epileptic medications and allopurinol, 
has also been attributed to a multitude of medications $[4,5]$ We describe here the second report of oxacillin related DRESS and the first associated with agranulocytosis. Given the barriers to confirming a definitive diagnosis in real-time, clinicians must maintain a high level of suspicion for this clinical syndrome. The mainstay of care is largely supportive management

\section{References:}

1. Jones TD, Jacobs LJ: The treatment of obstinate chorea with nirvanol. JAMA 1932; 99(1): 18-21

2. Muller $P$, Dubreil $P$, Mahé $A$ et al: Drug hypersensitivity syndrome in a WestIndian population. Eur J Dermatol, 2003; 13(5): 478-81

3. Kardaun SH, Sekula P, Valeyrie-Allanore L et al: Drug reaction with eosinophilia and systemic symptoms (DRESS): An original multisystem adverse drug reaction. Results from the prospective RegiSCAR study. Br J Dermatol, 2013; 169(5): 1071-80

4. Hübner ST, Bertoli R, Rätz Bravo AE et al: C-reactive protein and procalcitonin in case reports of drug reaction with eosinophilia and systemic symptoms (DRESS) syndrome. Int Arch Allergy Immunol, 2018; 176(1): 44-54

5. Renda F, Landoni G, Bertini Malgarini R et al: Drug reaction with eosinophilia and systemic symptoms (DRESS): A national analysis of data from 10-year post-marketing surveillance. Drug Saf, 2015; 38(12): 1211-18

6. Rabenkogo A, Vigue MG, Jeziorski E: [DRESS syndrome]. Arch Pediatr, 2015; 22(1): 57-62 [in French]

7. Cacoub $P$, Musette $P$, Descamps $V$ et al: The DRESS syndrome: $A$ literature review. Am J Med, 2011; 124(7): 588-97

8. James J, Sammour YM, Virata AR et al: Drug reaction with eosinophilia and systemic symptoms (DRESS) syndrome secondary to furosemide: Case report and review of literature. Am J Case Rep, 2018; 19: 163-70

9. Kardaun SH, Sidoroff A, Valeyrie-Allanore L et al: Variability in the clinical pattern of cutaneous side-effects of drugs with systemic symptoms: Does a DRESS syndrome really exist? Br J Dermatol, 2007; 156(3): 609-11

10. Hiransuthikul A, Rattananupong T, Klaewsongkram J et al: Drug-induced hypersensitivity syndrome/drug reaction with eosinophilia and systemic symptoms (DIHS/DRESS): 11 years retrospective study in Thailand. Allergol Int, 2016; 65(4): 432-38 with antihistamines and anti-inflammatory ointments, typically with resolution within days to weeks.

\section{Conflicts of interest}

None.

11. Chen YC, Chiu HC, Chu CY: Drug reaction with eosinophilia and systemic symptoms: A retrospective study of 60 cases. Arch Dermatol, 2010; 146(12): 1373-79

12. Lavenant $P$, Roue $J M$, Huet $F$ et al: [DRESS syndrome and agranulocytosis, a rare combination]. Arch Pediatr, 2017; 24(8): 752-56 [in French]

13. Kato $M$, Kano $Y$, Sato $Y$, Shiohara T: Severe agranulocytosis in two patients with drug-induced hypersensitivity syndrome/drug reaction with eosinophilia and systemic symptoms. Acta Derm Venereol, 2016; 96(6): 842-43

14. Roujeau JC, Allanore L, Liss $Y$ et al: Severe cutaneous adverse reaction to drugs (SCAR): Definitions, diagnostic criteria, genetic predisposition. Dermatol Sinica, 2009; 27: 203-9

15. Um SJ, Lee SK, Kim YH et al: Clinical features of drug-induced hypersensitivity syndrome in 38 patients. J Investig Allergol Clin Immunol, 2010; 20(7): 556-62

16. Funck-Brentano E, Duong TA, Bouvresse $S$ et al: Therapeutic management of DRESS: A retrospective study of 38 cases. J Am Acad Dermatol, 2015; 72(2): 246-52

17. Kirchhof MG, Wong A, Dutz JP: Cyclosporine treatment of drug-induced hypersensitivity syndrome. JAMA Dermatol, 2016; 152(11): 1254-57

18. Joly $P$, Janela B, Tetart F et al: Poor benefit/risk balance of intravenous immunoglobulins in DRESS. Arch Dermatol, 2012; 148(4): 543-44

19. Singer EM, Wanat KA, Rosenbach MA: A case of recalcitrant DRESS syndrome with multiple autoimmune sequelae treated with intravenous immunoglobulins. JAMA Dermatol, 2013; 149(4): 494-95 